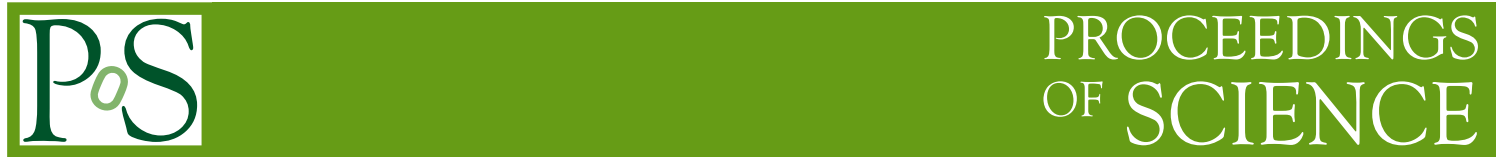

\title{
A jet model for black-hole X-ray sources
}

\author{
N. D. Kylafis*, I. E. Papadakis, P. Reig \\ University of Crete and \\ Foundation for Research and Technology - Hellas, Greece \\ kylafis@physics.uoc.gr
}

\begin{abstract}
A jet model for Galactic black-hole X-ray binaries will be presented that appears to explain several observational characteristics. In particular, it explains the energy spectrum from radio to hard $\mathrm{X}$-rays, the time-lags as a function of Fourier frequency, the increase of the variability amplitude (QPO and high frequency) with increasing photon energy, and the narrowing of the autocorrelation function with increasing photon energy. On the other hand, there are additional observational constraints that no model has tried to explain yet. It is important that we all try to address these constraints if we are to make any progress in understanding black-hole X-ray sources.
\end{abstract}

VI Microquasar Workshop: Microquasars and Beyond

September 18-22 2006

Società del Casino, Como, Italy

\footnotetext{
* Speaker.
} 


\section{Introduction}

We will be a little provocative in our contribution, not because we think that we have the right answers to outstanding questions regarding black-hole X-ray binaries (or even some of the right answers), but because we want to bring forward and stress some important observational conclusions (new and old) that most models do not address.

\subsection{Observational conclusion 1}

Miller et al. (2006) found that an observation of GX 339-4 revealed an accretion disk extending to the Inner Stable Circular Orbit (ISCO), when the source was in the low/hard state. The same is true for Swift J1753.5-0127 (Miller, Homan, and Miniutti 2006). We understand that the above conclusion is not universally accepted, as the common belief is that the accretion disk in the low/hard state is truncated at some inner radius larger than the ISCO. Nevertheless, it is accepted by the community that the data allow for such a conclusion.

If this conclusion is correct, then the radio jet that is seen in the low/hard state is not associated with a truncated accretion disk. This then, leads us to suggest that there is no reason to exclude a jet (weak as it may be) when the source is in the high/soft state and the accretion disk extends all the way in to the ISCO.

Even if the above conclusion is proven to be incorrect, we still suggest that a weak (undetected up to now) jet may be present when the black-hole X-ray source is in the high/soft state. We will support this idea below.

\subsection{Observational conclusion 2}

Fourier resolved spectroscopy has shown that the accretion disk is not variable on time scales characteristic of X-ray variability (i.e. less than about $100 \mathrm{~s}$ ), at least in three black-hole X-ray sources: Cyg X-1 (Revnivtsev, Gilfanov, and Churazov 1999; Gilfanov, Churazov, and Revnivtsev 2000; Churazov, Gilfanov, and Revnivtsev 2001), GX 339-4 (Revnivtsev, Gilfanov, and Churazov 2001), and 4U 1543-47 (Reig et al. 2006).

It is inescapable then, that the observed variability in hard $\mathrm{X}$ rays comes from the jet (or whatever you want to call that thing above and below the accretion disk, e.g., corona, wind, flares, etc.).

\subsection{Observational conclusion 3}

It has been shown by Pottschmidt et al. (2000) that the time-lags as a function of Fourier frequency are identical in the low/hard and the high/soft states. Thus, it is natural to assume that identical mechanisms must be producing the time-lags in both the low/hard and the high/soft states. This introduces significant constraints to models. In addition, if the jet introduces the time-lags in the low/hard state, then a weak jet must be present in the high/soft state.

\section{Jet model}

For whatever it is worth, the jet model proposed by Reig, Kylafis, and Giannios (2003) explains the time-lags in a natural way as time delays due to upscattering of soft photons in the jet. 


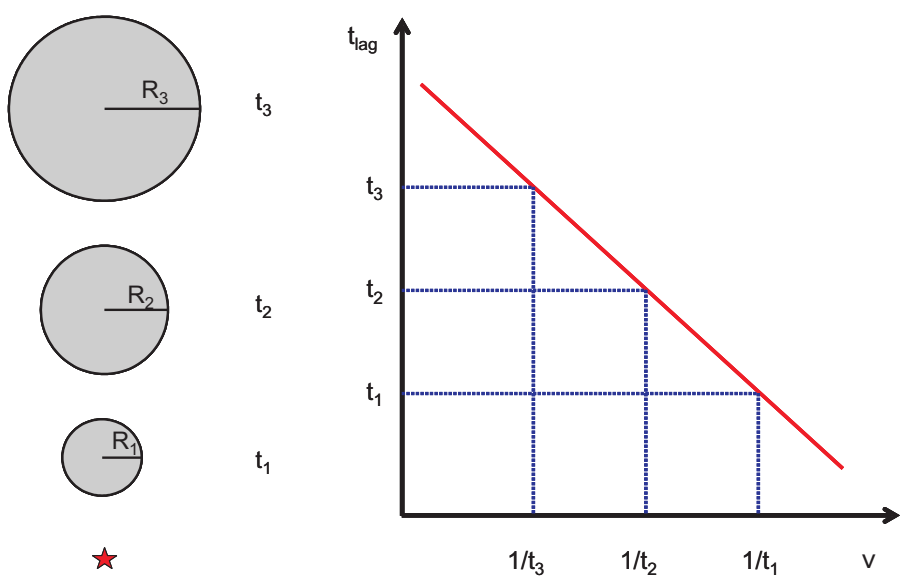

Figure 1: Schematic of a "jet" with properties discussed in the text.

To understand how the time-lags can be inversely proportional to Fourier frequency, consider the following simplified picture of the jet (Fig. 1). Let the jet consist of a series of spheres with increasing radii $R_{i}$. Arrange the densities of the spheres so that a soft photon from the source below (denoted by a $*$ in the Figure) has equal probability to scatter in any of the spheres. Let the time delay due to scattering in the sphere with radius $R_{i}$ be $t_{i}$. It is well known that Compton scattering that causes a delay $t_{i}$ acts as a filter and cuts off all frequencies higher that $\sim 1 / t_{i}$. In other words, the larger the Fourier frequency that is observed the smaller the time-lag.

The model of Reig et al. (2003) makes the assumption that the density in the jet varies inversely proportional with height. This ensures that the optical depth to electron scattering is the same per decade of height. Thus, if the total optical depth along the jet is of order unity, the soft input photons scatter with equal probability at all heights in the jet. Thus, all time lags from zero to a maximum value are sampled by the soft photons, but only frequencies less than the inverse time lag survive and are observed. A Monte Carlo calculation (Reig et al. 2003) has verified that the time lags are inversely proportional to Fourier frequency.

Two additional constraints regarding the flattening of the power spectrum at high frequencies and the narrowing of the autocorrelation function with increasing photon energy has also been explained with the jet model (Giannios, Kylafis, and Psaltis 2004).

Also, for XTE J1118+480, the only source for which we have simultaneous observations from radio to hard X-rays, the energy spectrum is reproduced extremely well over 10 orders of magnitude in energy by the jet model (Giannios 2005). It must be stressed here, that the energy spectra and the time variability are explained with the same values of the parameters of the model.

\section{What next?}

We are of the opinion that hardly any model in Astrophysics is entirely correct! Thus, we think that all models should be pushed to their limits. Some recent observational facts place very stringent constraints on all models. 
Pottschmidt et al. (2003) have fitted the power spectra of Cyg X-1 (over a period of four years) with four broad Lorentzians with peak frequencies $v_{1}, v_{2}, v_{3}$, and $v_{4}$.

\subsection{Unexplained observational fact 1}

The ratios of the peak frequencies of the above four Lorentzians are constant with time (see Fig. 5 of Pottschmidt et al. 2003). This means that the four Lorentzians "talk" to each other.

\subsection{Unexplained observational fact 2}

In Fig. 7a of Pottschmidt et al. (2003), one can see that the average time lags (in the $3.2-10$ $\mathrm{Hz}$ range) are correlated with the peak frequencies $v_{1}, v_{2}$, and $v_{3}$.

Similar correlations (Fig. 7b) have been found between the power-law spectral index $\Gamma$ and the peak frequencies $v_{1}, v_{2}$, and $v_{3}$.

It is not surprising then that $\Gamma$ is correlated (Fig. 7c) with the average time lags (in the $3.2-10$ Hz range).

\section{Summary}

In our opinion, the jet in black-hole X-ray binaries is not simply an ornament. It appears to be responsible not only for a large part of the energy spectrum (radio, optical, hard X-rays), but also for the observed time variability.

The three broad Lorentzians in the power spectrum with peak frequencies $v_{1}, v_{2}$, and $v_{3}$ seem to be produced by one mechanism (since their ratios are constant) and they "communicate" with the power-law spectral index $\Gamma$. In other words, each observed value of $\Gamma$ in Cyg X-1 is associated with specific values of the peak frequencies $v_{1}, v_{2}$, and $v_{3}$.

With such stringent constraints, life will be difficult for modelers, but no progress in our understanding will be made unless we address all the observational facts simultaneously. Addressing only the spectra or only the time lags or only some observational facts will not lead us anywhere.

Acknowledgments: This work has been supported in part by a PYTHAGORAS II grant from the Ministry of Education of Greece.

\section{References}

[1] Churazov, E., Gilfanov, M., Revnivtsev, M. 2001, MNRAS, 321, 759

[2] Giannios, D. 2005, A\&A, 437, 1007

[3] Giannios, D., Kylafis, N. D., Psaltis, D. 2004, A\&A, 425, 163

[4] Gilfanov, M., Churazov, E., Revnivtsev, M. 2000, MNRAS, 316, 923

[5] Miller, J.M., Homan, J., Miniutti, G. 2006, ApJ, 652, L113

[6] Miller, J.M., Homan, J., Steeghs, D., Rupen, M., Hunstead, R.W., Wijnands, R., Charles, P.A., Fabian A.C. 2006, ApJ, 653,525

[7] Pottschmidt, K., Wilms, J., Nowak, M. A., Heindl, W. A., Smith, D. M., Staubert, R. 2000, A\&A, 357, L17 
[8] Pottschmidt, K., Wilms, J., Nowak, M. A., Pooley, G. G., Gleissner, T., Heindl, W. A., Smith. D. M., Remillard, R., Staubert, R. 2003, A\&A, 407, 1039

[9] Reig, P., Kylafis, N. D., Giannios, D. 2003, A\&A, 403, L15

[10] Reig, P., Papadakis, I. E., Shrader, C. R., Kazanas, D. 2006, ApJ, 644, 424

[11] Revnivtsev, M., Gilfanov, M., Churazov, E. 1999, A\&A, 347, L23

[12] Revnivtsev, M., Gilfanov, M., Churazov, E. 2001, A\&A, 380, 520 